\title{
Long non-coding RNA HOTAIR is associated with human cervical cancer progression
}

\author{
HEE JUNG KIM ${ }^{1 *}$, DAE WOO LEE ${ }^{2,3^{*}}$, GA WON YIM ${ }^{1}$, EUN JI NAM ${ }^{1}$, \\ SUNGHOON KIM ${ }^{1}$, SANG WUN KIM ${ }^{1}$ and YOUNG TAE KIM ${ }^{1}$

\begin{abstract}
${ }^{1}$ Institute of Women's Life Medical Science, Division of Gynecologic Oncology, Department of Obstetrics and Gynecology, Yonsei University College of Medicine, Seoul; ${ }^{2}$ Department of Obstetrics and Gynecology, Yonsei University Graduate School, Seoul; ${ }^{3}$ Department of Obstetrics and Gynecology, Bucheon

St. Mary's Hospital, Catholic University College of Medicine, Bucheon, Republic of Korea
\end{abstract}

Received August 20, 2014; Accepted October 23, 2014

DOI: $10.3892 /$ ijo.2014.2758

\begin{abstract}
The functions of many long non-coding RNAs (lncRNAs) in human cancers remain to be clarified. The lncRNA Hox transcript antisense intergenic RNA (HOTAIR) has been reported to reprogram chromatin organization and promote breast and colorectal cancer metastasis, the involvement of lncRNAs in cervical cancer is just beginning to be studied. In the present study, we examined the expression and the functional role of HOTAIR in cervical cancer. HOTAIR expression was determined in cervical cancer tissues $(n=111)$ and corresponding normal tissues $(n=40)$ by using real-time polymerase chain reaction, and its correlation with clinical parameters and prognosis were analyzed. To determine the effect of HOTAIR knockdown and overexpression in cervical cancer cell lines, we used the CCK- 8 assay, wound healing migration and Matrigel invasion assay. The expression level of HOTAIR in cervical cancer tissues was higher than that in corresponding non-cancerous tissues. High HOTAIR expression correlated with lymph node metastasis, and reduced overall survival. A multivariate analysis showed that HOTAIR was a prognostic factor for predicting cervical cancer recurrence. Knockdown of HOTAIR reduced cell proliferation, migration, and invasion in cervical cancer cell lines. Moreover, HOTAIR regulated the expression of vascular endothelial growth factor,
\end{abstract}

Correspondence to: Dr Young Tae Kim, Institute of Women's Life Medical Science, Division of Gynecologic Oncology, Department of Obstetrics and Gynecology, Yonsei University College of Medicine, 50 Yonsie-ro, Seodaemungu, Seoul 120-752, Republic of Korea E-mail: ytkchoi@yuhs.ac

Abbreviations: lncRNAs, long non-coding RNAs; HOTAIR, Hox transcript antisense intergenic RNA; VEGF, vascular endothelial growth factor; MMP-9, matrix metalloproteinase-9; EMT, epithelialto-mesenchymal transition; miRNAs, microRNAs

${ }^{*}$ Contributed equally

Key words: HOTAIR, invasion, metastasis, prognosis, cervical cancer matrix metalloproteinase-9 and epithelial-to-mesenchymal transition (EMT)-related genes, which are important for cell motility and metastasis. Therefore, HOTAIR may promote tumor aggressiveness through the upregulation of VEGF and MMP-9 and EMT-related genes. These findings indicate that HOTAIR may represent a novel biomarker for predicting recurrence and prognosis and serve as a promising therapeutic target in cervical cancer.

\section{Introduction}

Non-coding RNAs (ncRNAs) are found in the genome of humans, mouse and other animals. However, the functions of ncRNAs are only partially understood. ncRNAs are mainly classified into housekeeping or regulatory ncRNAs (1-3). Based on transcript size, regulatory ncRNAs can be further grouped into 2 subclasses: small ncRNAs (20-200 nt) and long ncRNAs (lncRNAs, >200 nt). microRNAs (miRNAs) have been the most extensively investigated of the small ncRNAs, and estimates suggest that $>1,000$ miRNAs regulate up to $30 \%$ of all protein-encoding genes (4-7). Characterization of the functional and clinical significance of some ncRNAs has shown that they are key factors in gene regulation and influence normal and cancer cell phenotypes (4,8-10).

Recent data have demonstrated that $>3,000$ human long intervening non-coding RNAs (lincRNAs) and most long ncRNAs are associated with DNA-binding proteins such as chromatin-modifying complexes (11) and epigenetically regulate the expression of multiple genes $(12,13)$. Transcription of lncRNAs has been shown to modulate gene activity in response to external oncogenic stimuli and DNA damage (14). This finding indicates the potential involvement of lncRNAs in the pathogenesis of human diseases, most notably in cancer (15). HOX transcript antisense intergenic RNA (HOTAIR) is a 2158-bp lncRNA that was identified from a custom tiling array of the HOXC gene cluster. Interaction of HOTAIR with the polycomb repressive complex 2 (PRC2), which is composed of EZH2, SUZ12 and EED, leads to the trimethylation of histone H3 lysine 27 and establishment of the repressive H3K27me3 chromatin mark (11). HOTAIR has been shown to inhibit tumor suppressor genes such as HOXD10, PGR, and the proto- 
Table I. Association between HOTAIR expression and clinicopathologic factors in cervical cancer $(\mathrm{n}=111)$.

\begin{tabular}{|c|c|c|c|c|}
\hline & \multirow[b]{2}{*}{$\mathrm{n}(\%)$} & \multicolumn{2}{|c|}{ HOTAIR expression } & \multirow[b]{2}{*}{ P-value } \\
\hline & & Low & High & \\
\hline Age $($ mean \pm SD $)$ & 111 & $50.4 \pm 2.51$ & $50.8 \pm 1.29$ & 0.8809 \\
\hline Stage & & & & 0.7671 \\
\hline $\mathrm{I}$ & $43(38.74)$ & 10 & 33 & \\
\hline II & $56(50.45)$ & 10 & 46 & \\
\hline III-IV & $12(10.81)$ & 2 & 10 & \\
\hline Cell type & & & & 0.2334 \\
\hline $\mathrm{SCC}$ & $78(70.27)$ & 17 & 61 & \\
\hline Adeno & $24(21.62)$ & 2 & 22 & \\
\hline Mixed & $3(2.7)$ & 1 & 2 & \\
\hline Other & $6(5.41)$ & 2 & 4 & \\
\hline Tumor size (cm) & & & & 0.8839 \\
\hline$<4$ & $66(60)$ & 14 & 52 & \\
\hline$\geq 4$ & $44(40)$ & 8 & 36 & \\
\hline Lymphatic invasion & & & & 0.6351 \\
\hline Yes & $58(52.25)$ & 10 & 48 & \\
\hline No & $53(47.75)$ & 12 & 41 & \\
\hline Lymph node metastasis & & & & 0.0437 \\
\hline Yes & $35(31.53)$ & 3 & 32 & \\
\hline No & $76(68.47)$ & 19 & 57 & \\
\hline
\end{tabular}

${ }^{a}$ Chi-square test or Fisher's exact test were used to calculate P-values. Adeno, adenocarcinoma; SCC, squamous cell carcinoma.

cadherin gene family in breast cancer cells (16). HOTAIR is a negative prognostic factor for breast, liver, colon, pancreatic and cervical cancer (17-19). Furthermore, increased HOTAIR expression has been correlated with enhanced breast and colon cancer metastasis. Although HOTAIR has been shown to play a critical role in the progression of breast, liver, colon and pancreatic cancers, little is known about the molecular mechanisms in cervical cancer.

Cervical cancer is the third most common cancer and the fourth leading cause of cancer death in women worldwide (20). Widespread implementation of Pap smear screening programs in recent years has decreased the incidence and mortality of cervical cancer in many countries (21). Despite this, cervical cancer continues to be a major public health problem (21). Cancer cell motility and invasion play a crucial role in the mortality of cervical cancer patients (22). Therefore, major research efforts have focused on the identification of tumor-specific markers for predicting the biological behavior of cervical cancers. Several miRNAs, including miR-214, miR-143, miR-375, miR-23b and miR-20, have been shown to modulate cervical cancer cell motility and invasion; these may represent potential prognostic markers for predicting the aggressiveness of cervical cancer (23-27). Increased understanding of the molecular mechanisms underlying cervical carcinogenesis and progression is required to identify reliable prognostic markers associated with tumor aggressiveness.

In the present study, we determined the expression and clinical significance of HOTAIR in cervical cancer. We found that HOTAIR was highly expressed in cervical cancer and was associated with disease recurrence. Furthermore, HOTAIR knockdown inhibited proliferation, migration and invasion of human cervical cancer cell lines. Also, we examined the molecular events that occur downstream of HOTAIR involvement in cervical cancer migration and invasion. These findings provide novel insights into the role of HOTAIR in the metastatic progression of cervical cancer.

\section{Materials and methods}

Human tissues. Cervical cancer samples were obtained from 111 female patients who underwent surgery at Yonsei Severance Hospital, Yonsei University, between 2007 and 2012. Specimens from patients with newly diagnosed invasive [FIGO (International Federation of Gynecology and Obstetrics) stage IA-IVB] cervical cancer who had not received prior treatment were included in the study. Forty samples of normal cervix from patients undergoing simple hysterectomy because of uterine leiomyomata were obtained as controls. Specimens from patients with concomitant gynecological cancer were excluded from the study. All specimens were immediately frozen in liquid nitrogen and stored at $-80^{\circ} \mathrm{C}$ until RNA extraction. The study was conducted according to the principles in the Declaration of Helsinki and was approved by the ethical committee of Yonsei Severance Hospital. Informed consent was obtained from all patients. The clinical information is summarized in Table I. 
Cell culture. SiHa (squamous cervical carcinoma), HeLa (epitheloid cervical carcinoma) and Caski (epidermoid cervical carcinoma established from a metastasis in the small bowel mesentery) human cervical cancer cell lines obtained from the American Type Culture Collection (ATCC, Rockville, MD, USA). SiHa and HeLa cells were cultured in Dulbecco's modified Eagle's medium, and Caski cells were cultured in RPMI-1640 medium (Gibco-BRL, Gaithersburg, MD, USA). The human keratinocyte cell line HaCaT was cultured in RPMI-1640 medium. The culture media were supplemented with $10 \%$ (vol/vol) fetal bovine serum and penicillin/streptomycin. The cell lines were maintained at $37^{\circ} \mathrm{C}$ in a humidified atmosphere of $5 \% \mathrm{CO}_{2}$ and $95 \%$ air. Cells with a passage number $<20$ were used in all experiments.

Quantitative real-time polymerase chain reaction ( $q R T-P C R)$. Total RNA was extracted from cancerous/non-cancerous specimens or cell lines using TRIzol ${ }^{\circledR}$ reagent (Invitrogen, Carlsbad, CA, USA), and $2 \mu \mathrm{g}$ of total RNA was reverse transcribed into first-strand cDNA by using a reverse transcription reagent kit (Invitrogen) according to the manufacturer's protocol. qRT-PCR was performed using the SYBR ${ }^{\circledR}$ Green real-time PCR kit (Toyobo, Co., Ltd., Osaka, Japan) in a 20- $\mu 1$ reaction volume, which contained $10 \mu \mathrm{l}$ of SYBR-Green Master PCR Mix, 5 pmole each of forward and reverse primers, $1 \mu 1$ of diluted cDNA template, and appropriate amounts of sterile distilled water. Conditions for the amplification of genes were as follows: initial denaturation at $95^{\circ} \mathrm{C}$ for $3 \mathrm{~min} ; 40$ cycles of denaturation at $95^{\circ} \mathrm{C}$ for $15 \mathrm{sec}$, annealing at $60^{\circ} \mathrm{C}$ for $60 \mathrm{sec}$, and elongation at $72^{\circ} \mathrm{C}$ for $60 \mathrm{sec}$; and final elongation at $72^{\circ} \mathrm{C}$ for $5 \mathrm{~min}$. qRT-PCR was performed on the ABI StepOnePlus Real-Time PCR system (Applied Biosystems, Foster City, CA, USA). All quantifications were performed with $U 6$ as the internal standard. The PCR primer sequences were as follows: HOTAIR, 5'-GGTAGAAAAAGCAACCAC GAAGC-3' (sense) and 5'-ACATAAACCTCTGTCTGTGAG TGCC-3' (antisense); E-cadherin, 5'-ATTCTGATTCTGC TGCTCTTG-3' (sense) and 5'-AGTAGTCATAGTCCTGGTC CT-3' (antisense); $\beta$-catenin, 5'-TGCAGTTCGCCTTCACT ATG-3' (sense) and 5'-ACTAGTCGTGGAATGGCACC-3' (antisense); vimentin, 5'-TGGATTCACTCCCTCTGGTT-3' (sense) and 5'-GGTCATCGTGATGCTGAGAA-3' (antisense); snail, 5'-GAGGCGGTGGCAGACTAG-3' (sense) and 5'-GAC ACATCGGTCAGACCAG-3' (antisense); twist, 5'-CGGGAG TCCGCAGTCTTA-3' (sense) and 5'-TGAATCTTGCTCAG CTTGTC-3' (antisense); and U6, 5'-CTCGCTTCGGCAGC ACA-3' (sense) and 5'-AACGCTTCAGGAATTTGCGT-3' (antisense). Relative gene expression was analyzed using the $2^{-\triangle \Delta C T}$ method, and the results were expressed as extent of change with respect to control values. qRT-PCR experiments were replicated at least 3 times.

Small interfering RNA (siRNA) transfection. HOTAIR siRNA (siHOTAIR-1 and siHOTAIR-2) and negative control siRNA (siNC) were purchased from Bioneer (Daejeon, Korea). Cells $\left(5 \times 10^{4}\right.$ cells/well) were seeded into 6-well plates and were transfected with $10 \mathrm{nM}$ siRNA in phosphate-buffered saline (PBS) using the G-Fectin kit (Genolution Pharmaceuticals Inc., Seoul, Korea) according to the manufacturer's protocol. These siRNA-transfected cells were used in the in vitro assays
$48 \mathrm{~h}$ post-transfection. The target sequences for HOTAIR siRNAs were as follows: siRNA-1, 5'-UUUUCUACCAGGUC GGUAC-3' and siRNA-2, 5'-AAUUCUUAAAUUGGGCU GG-3'.

Plasmid constructs and the generation of stable cell line. The human HOTAIR transcript variant 3 cDNA was amplified by PCR and was inserted into the pLenti6/V5-D-TOPO vector according to ViraPower ${ }^{\mathrm{TM}}$ Lentiviral Expression systems (Invitrogen). Briefly, plasmid was transfected into the 293FT cell line and then lentivirus was infected in desired cell line. Selection of HOTAIR stable transfected cells was performed in medium containing blasticidin (Invitrogen).

Cell proliferation assay. Cell proliferation was evaluated using the Cell Counting Kit-8 (CCK-8) assay (Dojindo Laboratories, Kumamoto, Japan). Cells $\left(2 \times 10^{3}\right.$ cells/well $)$ were seeded into 96-well flat-bottomed plates in $100 \mu \mathrm{l}$ of complete medium. The cells were incubated overnight to allow for cell attachment and recovery and were then transfected with siNC or siHOTAIR for $24,48,72$ and $96 \mathrm{~h}$. CCK-8 solution $(10 \mu \mathrm{l})$ was added to each well, and the cells were incubated for an additional $2 \mathrm{~h}$. Absorbance was measured at $450 \mathrm{~nm}$ using a microplate reader. Three independent experiments were performed in triplicate.

Matrigel invasion assay. The Matrigel invasion assay was performed using the BD BioCoat Matrigel Invasion Chamber (pore size: $8 \mathrm{~mm}, 24$-well; BD Biosciences, Bedford, MA, USA) according to the manufacturer's protocol. siHOTAIRtransfected cells and siNC-transfected cells $\left(5 \times 10^{4}\right.$ cell/plate $)$ were plated in the upper chamber in serum-free medium, and complete medium was added to the bottom chamber. The Matrigel invasion chamber was incubated for $48 \mathrm{~h}$ at $37^{\circ} \mathrm{C}$ under $5 \% \mathrm{CO}_{2}$. Non-invading cells were removed from the upper chamber using cotton-tipped swabs. Cells that had invaded through the pores onto the lower side of the filter were stained (Diff-Quik; Sysmex, Kobe, Japan), and these were counted using a hemocytometer. The number of invaded siHOTAIR-transfected cells was expressed as fold-change relative to siNC-transfected cells, which was set at 1 . The assay was replicated at least 3 times.

Wound healing migration assay. Cells transfected with siNC or siHOTAIR $\left(5 \times 10^{5}\right.$ cells/well $)$ were seeded into 6 -well culture plates with serum-containing medium and were cultured until the cell density reached $\sim 90 \%$ confluence. The serum-containing medium was removed, and cells were serum starved for $24 \mathrm{~h}$. When the cell density reached $\sim 100 \%$ confluence, an artificial homogeneous wound was created by scratching the monolayer with a sterile $200-\mu 1$ pipette tip. After scratching, the cells were washed with serum-free medium. Images of cells migrating into the wound were captured at 0 , 24 and $48 \mathrm{~h}$ using a microscope. The assay was performed in triplicate.

Western blot analysis. Cells were transfected with siNC or siHOTAIR for $48 \mathrm{~h}$, washed with ice-cold 0.01 M PBS (pH 7.2), and lysed in lysis buffer [50 mM Tris- $\mathrm{HCl}$ (pH 7.4), $150 \mathrm{mM}$ saline, $1 \%$ Nonidet P-40, and $0.1 \%$ sodium dodecyl sulfate 

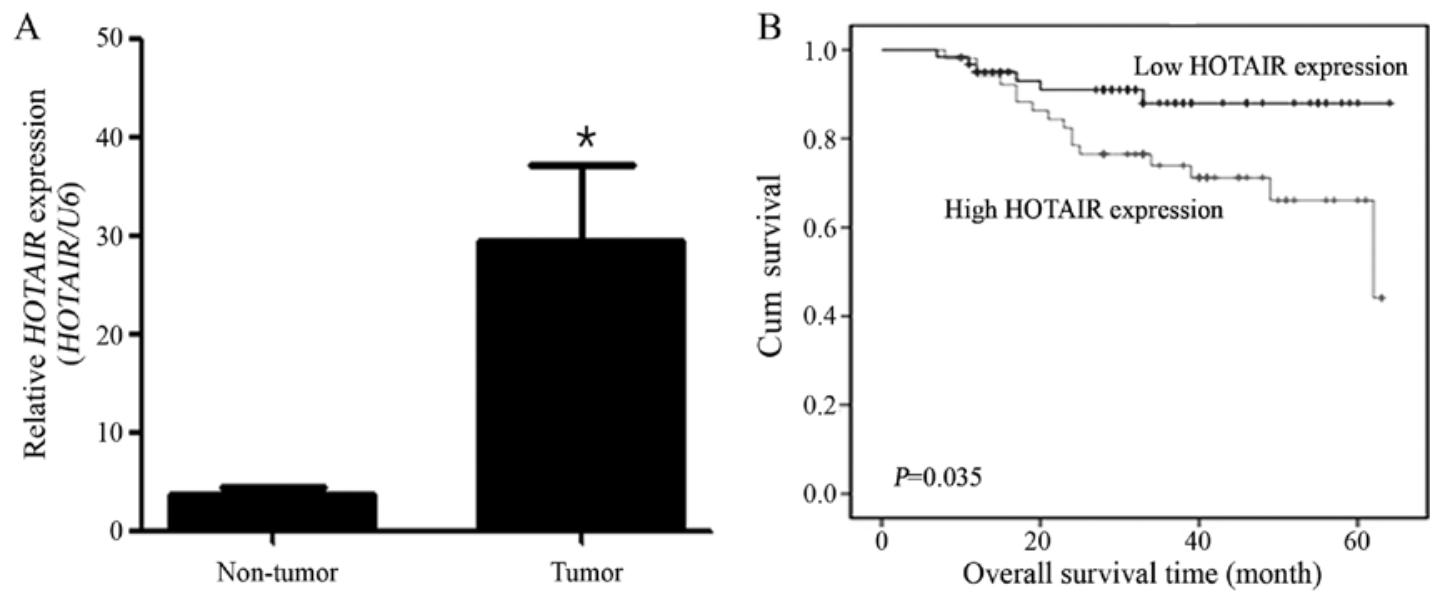

Figure 1. Relative HOTAIR expression and its clinical significance. (A) HOTAIR expression was significantly higher in cervical cancer tissues (n=111) than in non-cancerous tissues $(\mathrm{n}=40)$. Relative HOTAIR expression was determined using qRT-PCR with $U 6$ as an internal control. Data are expressed as mean \pm SD. ${ }^{*} \mathrm{P}<0.05$ vs. non-tumor control. (B) Kaplan-Meier overall survival curves of the patients with cervical cancer and different levels of HOTAIR (log-rank test; $\mathrm{P}=0.035)$.

(SDS)] supplemented with protease inhibitors. Protein concentrations were determined using Bio-Rad protein assay reagent according to the Bradford method (Bio-Rad Laboratories, Hercules, CA, USA). Samples were boiled for $5 \mathrm{~min}$, subjected to $10 \%$ SDS-PAGE, and transferred electrophoretically to polyvinylidene difluoride membranes (Millipore, Billerica, MA, USA). Membranes were blocked with 5\% non-fat dried milk in $1 \mathrm{X}$ Tris-buffered saline containing $0.1 \%$ Tween-20 (TBST; pH 7.6) at room temperature for $1 \mathrm{~h}$ and were then incubated with primary antibody at $4^{\circ} \mathrm{C}$ overnight under constant agitation. The primary antibodies used included: rabbit anti-human VEGF (1:500 dilution; Abcam, Cambridge, MA, USA), rabbit anti-human MMP-9 (1:1,000 dilution; Cell Signaling Technology, Beverly, MA, USA), rabbit anti-human E-cadherin (1:1,000 dilution; Cell Signaling Technology), rabbit anti-human $\beta$-catenin (1:1,000 dilution; Cell Signaling Technology), mouse anti-human Vimentin (1:1,000 dilution; Sigma, St. Louis, MO, USA), mouse anti-human Snail (1:1,000 dilution; Cell Signaling Technology), rabbit anti-human Twist (1:1,000 dilution; Abcam), and mouse anti-human $\beta$-actin antibody (1:5,000 dilution; Sigma). Membranes were washed 3 times with $1 \mathrm{X}$ TBST, incubated with a horseradish peroxidaseconjugated anti-rabbit secondary antibody (1:2,000 dilution; Abcam) or anti-mouse secondary antibody (1:2,000 dilution; Abcam) for $1 \mathrm{~h}$ at room temperature under constant agitation, and then washed 3 times with 1X TBST. Proteins were visualized using an enhanced chemiluminescence system (ECL ${ }^{\mathrm{TM}}$; Amersham, Little Chalfont, UK), and band intensities were quantified using the Luminescent image analyzer (LAS 4000 mini; Fujifilm, Uppsala, Sweden).

Statistical analysis. SPSS software (standard version 20.0; IBM) was used for all statistical analyses. Data are expressed as the mean \pm standard deviation (SD). The association between HOTAIR expression and clinicopathological characteristics was assessed using the Pearson's $\chi^{2}$ test, Student's t-test, and Fisher's exact test. Overall survival was analyzed by the Kaplan-Meier method, and the differences between groups were estimated by the log-rank test. Multivariate survival analysis was performed for the significant parameters in the univariate analysis using the stepwise Cox regression model analysis. All statistical tests were two-sided, and $\mathrm{P}<0.05$ was considered to indicate a statistically significant result.

\section{Results}

Association between HOTAIR expression and clinicopathologic factors in cervical cancer. The expression of HOTAIR lncRNA was determined in cervical cancer tissues $(\mathrm{n}=111)$ and corresponding normal tissues ( $\mathrm{n}=40)$ using qRT-PCR. HOTAIR expression in cervical cancer tissues was $>30$-fold that in noncancerous tissues (Fig. 1A), suggesting that the expression of HOTAIR is upregulated in cervical cancer. To evaluate the prognostic value of HOTAIR for predicting clinical outcome in cervical cancer, HOTAIR expression levels were determined in an independent panel consisting of 111 cervical cancer patients with extensive clinical follow-up (Table I). The patients were divided into low $(\mathrm{n}=22)$ and high $(\mathrm{n}=89)$ HOTAIR expression groups, and clinicopathologic features were compared between the two groups. Age, stage, cell type and lymphatic invasion were not significantly different between the low and high HOTAIR expression groups. In contrast, HOTAIR expression was correlated with lymph node metastasis $(\mathrm{P}=0.0437)$. Multivariate Cox regression model analysis was performed to further evaluate the prognostic significance of HOTAIR expression and clinicopathologic characteristics on recurrence (Table II). HOTAIR expression was a significant prognostic indicator for recurrence in cervical cancer patients (relative risk=5.281; $\mathrm{P}=0.0493$ ). As shown in Fig. 1B, HOTAIR expression levels were correlated with overall survival HOTAIR (log-rank test; $\mathrm{P}=0.035)$. These data suggest that HOTAIR expression represent an independent prognostic factor for survival and that the overexpression of HOTAIR might play an important role in the program of cervical cancer.

HOTAIR knockdown decreases cell proliferation in cervical cancer cells. To determine the functional role of HOTAIR in cervical cancer, siRNA was used to downregulate HOTAIR 

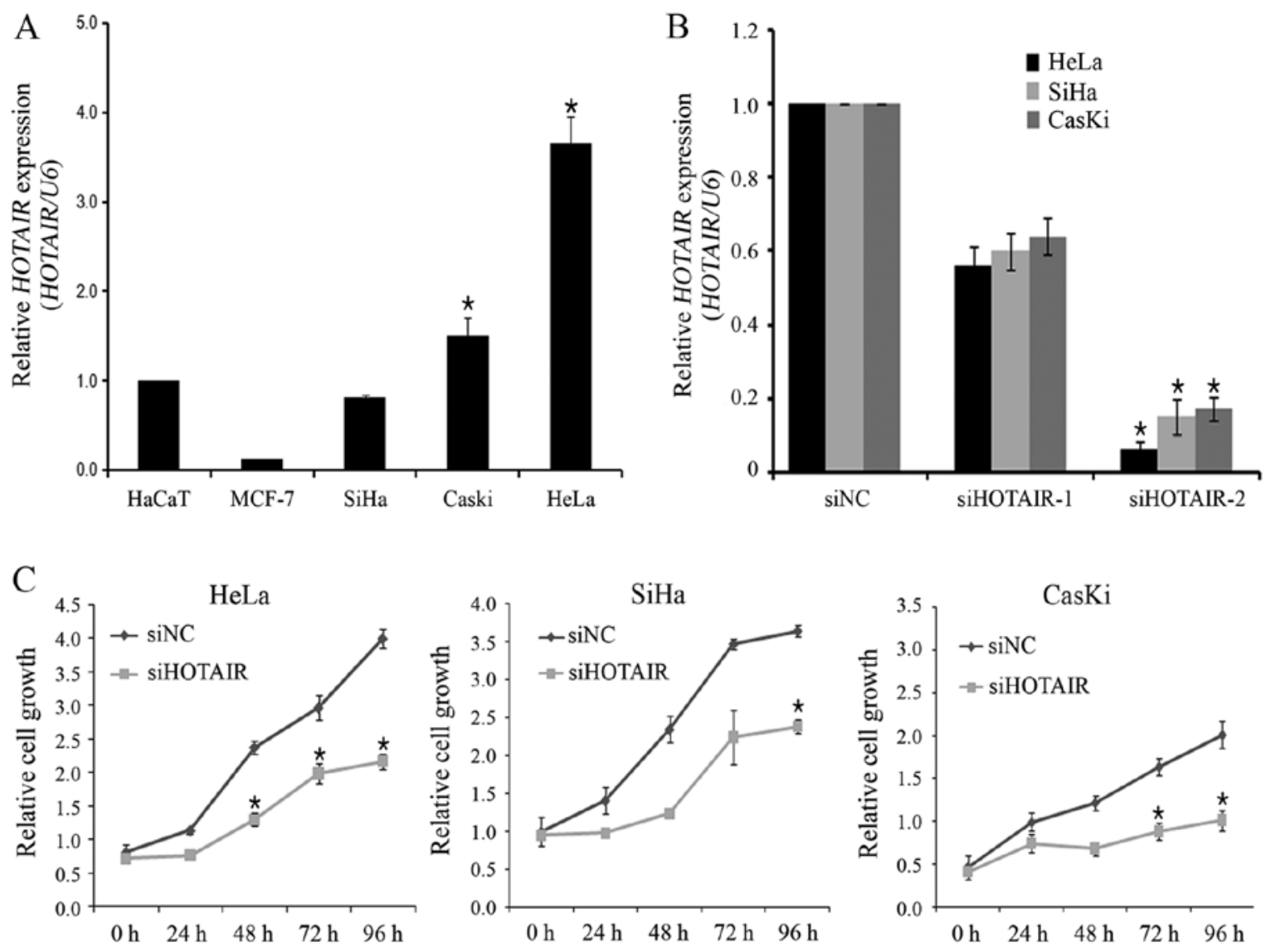

Figure 2. Knockdown of HOTAIR inhibits the cell proliferation of cervical cancer cells. (A) Expression of HOTAIR in cervical cancer cells. HOTAIR expression was evaluated using qRT-PCR with $U 6$ as an internal control. (B) Cells were transfected with HOTAIR-specific siRNA and negative control siRNA (siNC), and knockdown efficiency was determined by qRT-PCR analysis. (C) Knockdown of HOTAIR decreases cell proliferation in HeLa, SiHa and CasKi cells. The proliferation of cervical cancer cells transfected with siHOTAIR and negative control siRNA (siNC) was determined using the CCK-8 assay. Bars indicate mean $\pm \mathrm{SD}$ of three independent experiments performed in triplicate. ${ }^{*} \mathrm{P}<0.05 \mathrm{vs}$. siNC.

Table II. Multivariate analysis for recurrence in cervical cancer patients.

\begin{tabular}{lccr}
\hline & \multicolumn{2}{c}{ Recurrence } \\
\cline { 2 - 4 } Factor & HR & $95 \%$ CI & P-value \\
\hline HOTAIR (Low vs. high) & 5.281 & $1.005-27.742$ & 0.0493 \\
Age & 0.949 & $0.907-0.993$ & 0.024 \\
Stage (I vs. II) & 0.484 & $0.148-1.582$ & 0.2298 \\
Stage (I vs. III-IV) & 2.428 & $0.484-12.168$ & 0.2807 \\
Cell type (SCC vs. adeno) & 2.288 & $0.768-6.819$ & 0.1375 \\
Cell type (SCC vs. mixed) & 44.548 & $8.469-234.335$ & $<0.001$ \\
Cell type (SCC vs. other) & 4.607 & $0.906-23.411$ & 0.0655 \\
Tumor size (<4 vs. $\geq 4$ cm) & 1.651 & $0.529-5.152$ & 0.3876 \\
Lymphatic invasion (Yes vs. no) & 0.974 & $0.391-2.426$ & 0.9543 \\
Lymph node metastasis (Yes vs. no) & 0.824 & $0.265-2.561$ & 0.7384 \\
\hline
\end{tabular}

Adeno, adenocarcinoma; SCC, squamous cell carcinoma; HR, hazard ratio; CI, confidence interval.

expression. For this, HOTAIR expression in SiHa, Caski and HeLa cervical cancer cell lines was first determined using qRT-PCR. As shown in Fig. 2A, HOTAIR expression levels were higher in HeLa cells than in SiHa and Caski cells. Therefore, HeLa cells were used for siRNA-mediated knock- down of HOTAIR expression. The knockdown efficiency of the 2 HOTAIR-specific siRNAs (siHOTAIR-1 and siHOTAIR-2) was evaluated, and siHOTAIR-2 was found to have higher silencing efficiency than siHOTAIR-1 did (Fig. 2B). Therefore, siHOTAIR-2 was selected for use in the subsequent in vitro 
A
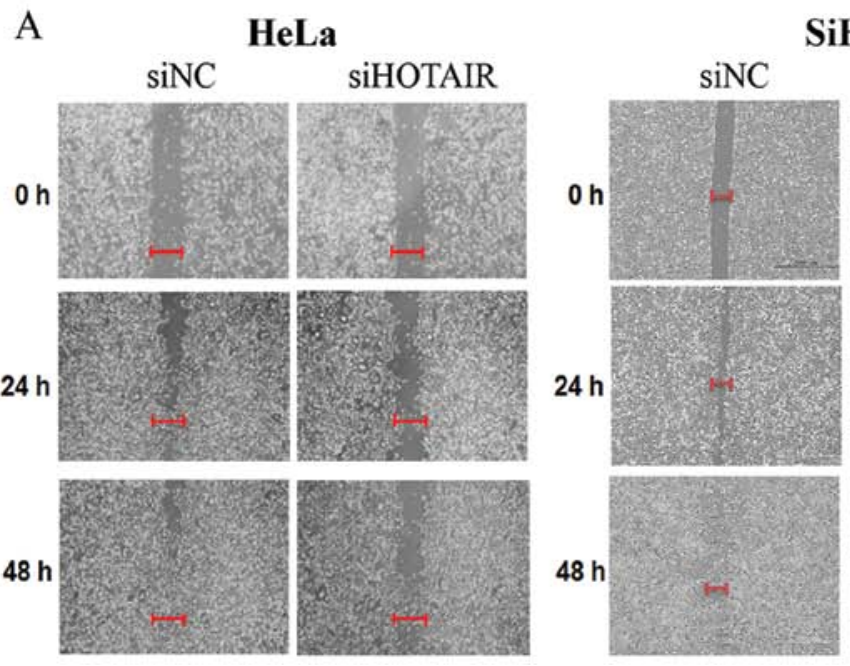

SiHa
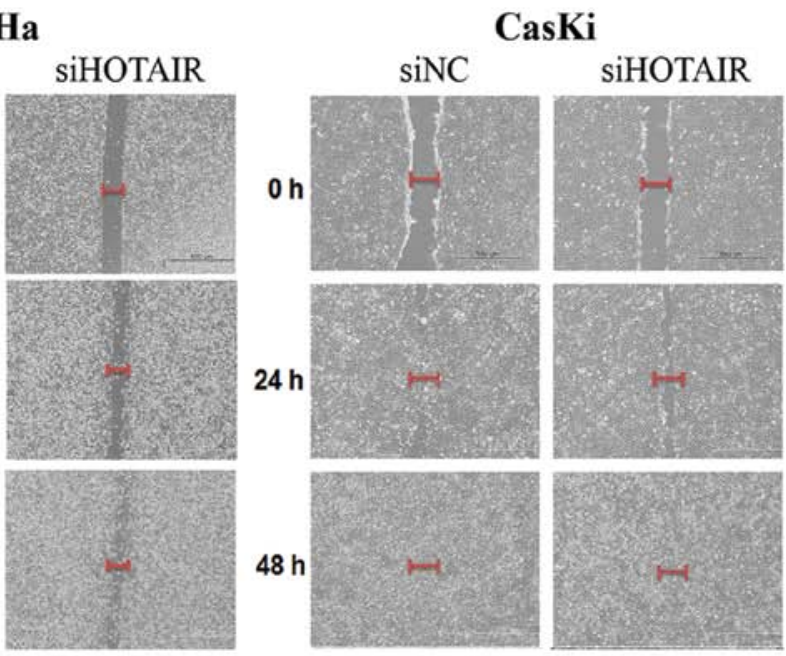

B

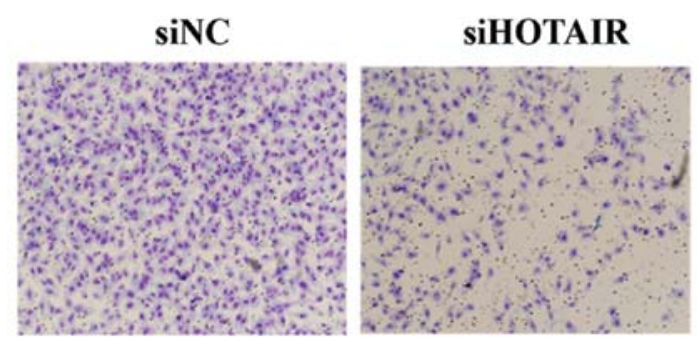

C
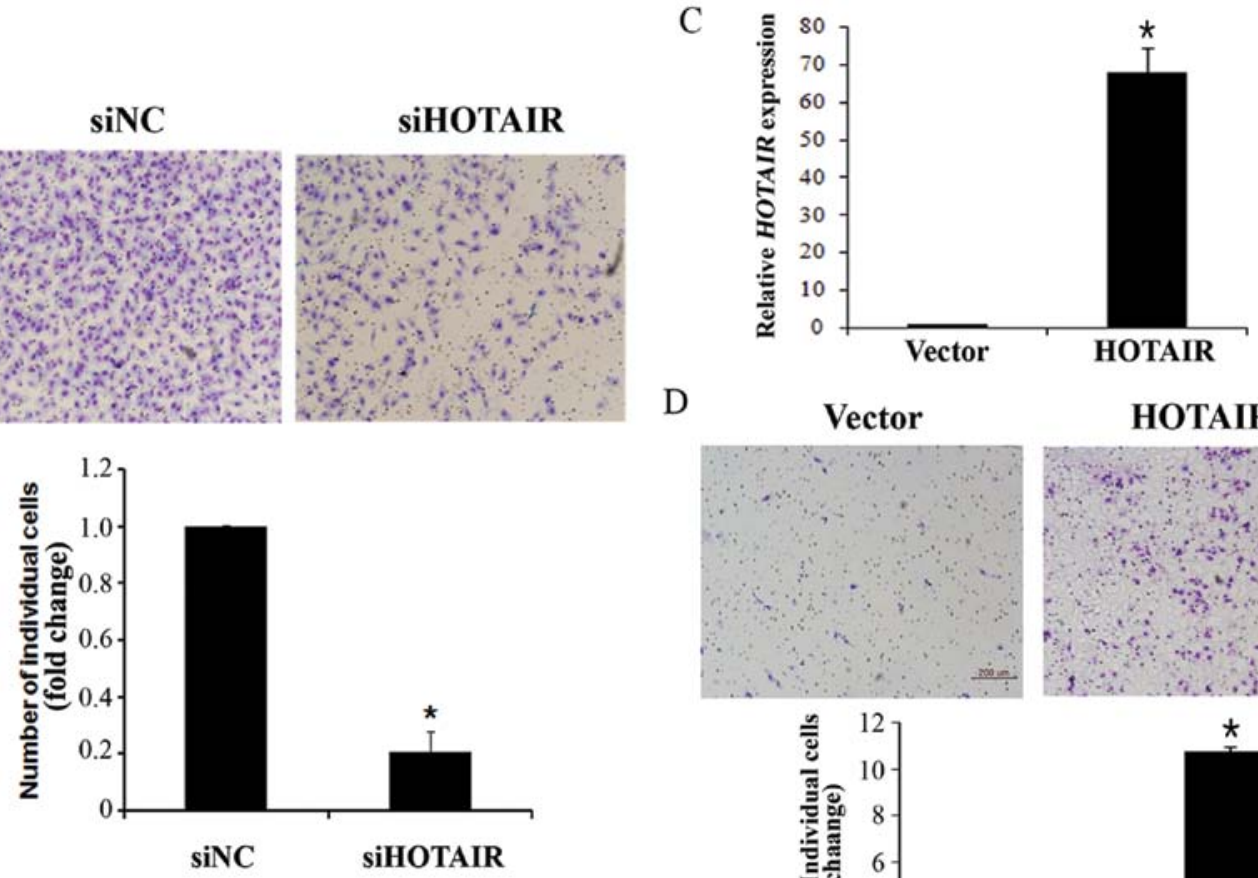

$\mathrm{D}$

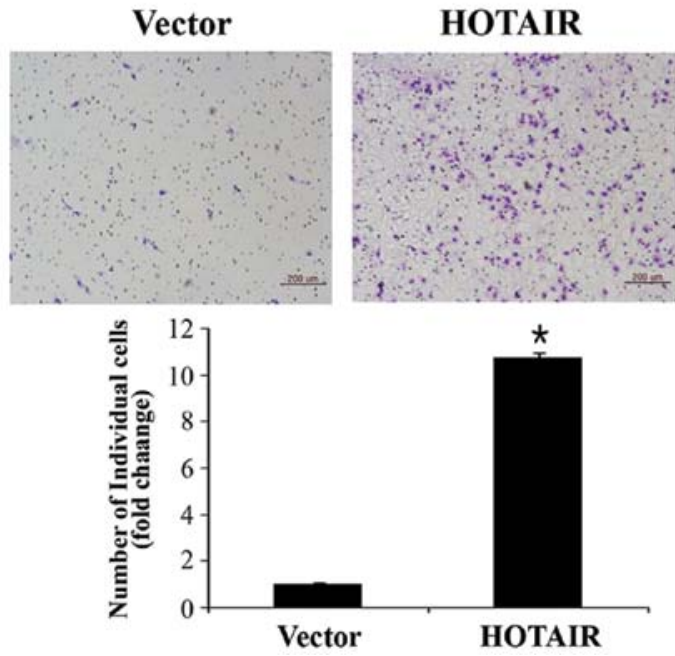

Figure 3. HOTAIR knockdown inhibits the migration and invasion of cervical cancer cells. (A) Wound healing assay was used to determine migration in siHOTAIR-transfected HeLa SiHa and CasKi cells (magnification, x200). (B) Matrigel invasion assay was used to determine invasion after $48 \mathrm{~h}$ in HeLa cells. (C) Overexpression of HOTAIR in SiHa cells analyzed by qRT-PCR. (D) Cell invasion was evaluated using Matrigel invasion chamber. Overexpression of HOTAIR in SiHa cells increased the invasive capacity after $48 \mathrm{~h}$. Each assay was performed in triplicate. Data are mean $\pm \mathrm{SD}$. ${ }^{*} \mathrm{P}<0.05$ vs. siNC.

biological assays. To determine the role of HOTAIR in cervical cancer cell growth, siHOTAIR-transfected cells were used in the CCK-8 assay. siRNA-mediated knockdown of HOTAIR decreased cell proliferation by $30 \%$ at $96 \mathrm{~h}$ post-transfection in HeLa cells (Fig. 2C). Also, HOTAIR siRNA inhibited cell proliferation in SiHa and Caski cells. This finding indicates that HOTAIR is involved in the proliferation of cervical cancer cells.

HOTAIR promotes cervical cancer cell migration and invasion. To investigate the effect of HOTAIR on migra- tion and invasion, siHOTAIR-transfected cells were used in wound healing and Matrigel invasion assays, respectively. The width of the wound closure was larger in siHOTAIRtransfected cells than in siNC-transfected of HeLa, SiHa and Caski cells (Fig. 3A). Therefore, downregulation of HOTAIR decreased the migration of cervical cancer cells. We also tested whether HOTAIR knockdown has an inhibitory effect on HeLa cell invasion. Knockdown of HOTAIR inhibited HeLa cell invasion $>80 \%$ (Fig. 3B). To further assess the role of HOTAIR in the pathogenesis of cervical cancer, SiHa cell lines stably expressing ectopic HOTAIR 
A

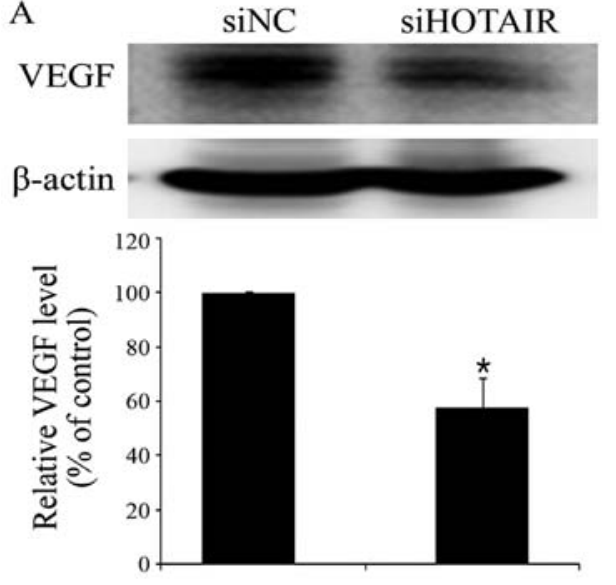

C

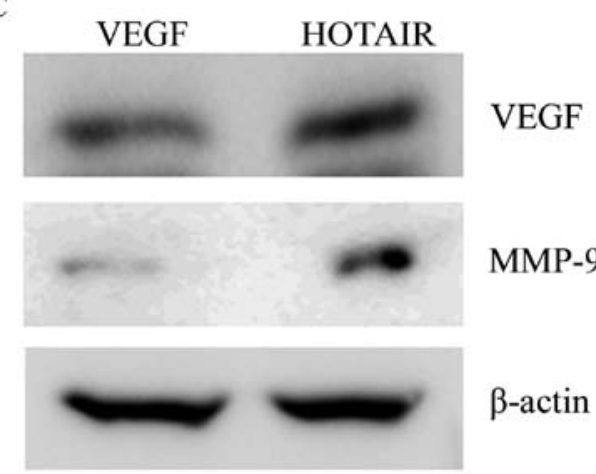

B
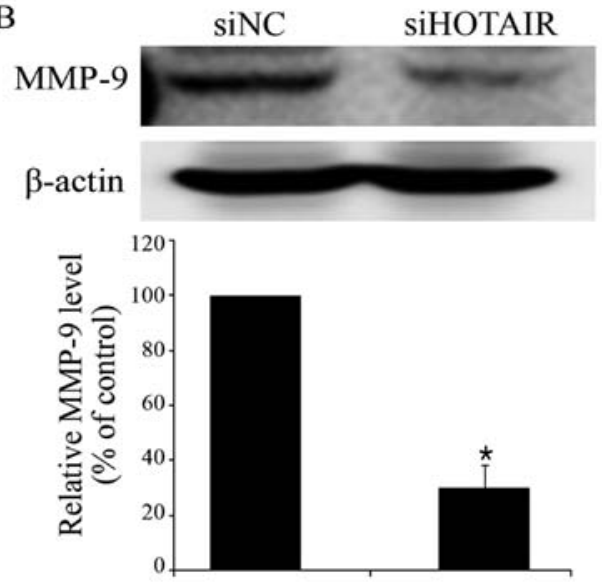

$\mathrm{D}$

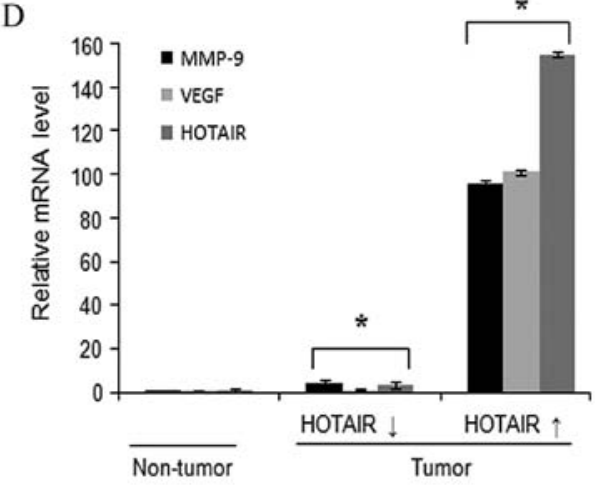

Figure 4. HOTAIR increases VEGF and MMP-9 expression in cervical cancer cells. Protein lysates were obtained from siHOTAIR and siNC-transfected HeLa cells $48 \mathrm{~h}$ post-transfection. (A) VEGF and (B) MMP-9 expression were analyzed by western blotting. (C) VEGF and MMP-9 levels were analyzed by western blotting in HOTAIR overexpression SiHa cells. Band intensities were quantitated, and VEGF and MMP-9 expression were normalized to that of $\beta$-actin. (D) VEGF and MMP-9 levels were determined by qRT-PCR in low groups and high HOTAIR expression groups of cervical cancer tissues. Each assay was performed in triplicate. Data are mean $\pm \mathrm{SD}$. ${ }^{*} \mathrm{P}<0.05$ vs. siNC.

were established (Fig. 3C). Consistent with the previous results, stable HOTAIR overexpression in SiHa cells resulted in a significantly increase the invasion ability of SiHa cells (Fig. 3D). Collectively, these results indicate that HOTAIR has an important role in the migratory and invasive phenotype of cervical cancer cells.

HOTAIR upregulates VEGF and MMP-9 expression in cervical cancer cells. VEGF and MMP-9 play an important role in tumor progression by promoting migration and invasion $(28,29)$. Therefore, the effect of HOTAIR on the expression levels of these proteins was determined in HeLa cells. VEGF and MMP-9 protein expressions were significantly lower in siHOTAIR-transfected cells than in siNC-transfected cells (Fig. 4A and B). In contrast, HOTAIR overexpression in SiHa cells promoted VEGF and MMP-9 protein expression (Fig. 4C). In addition, the high expression level of HOTAIR in cervical cancer tissues associated with upregulation of VEGF and MMP-9 expression levels compared with the low expression groups (Fig. 4D). Taken together, our findings suggest that HOTAIR may promote cervical cancer cell migration and invasion through the upregulation of VEGF and MMP-9 expression.

Inhibition of HOTAIR reversed EMT-related genes in cervical cancer cells. Because the EMT is important in cell migration and invasion, we also investigated whether direct inhibition of HOTAIR could reverse EMT-related markers in HeLa cells using real-time RT-PCR and western blot assays following HOTAIR knockdown. As anticipated, the siHOTAIR resulted in an increase in the expression of E-cadherin and a decrease in the expression of $\beta$-catenin and vimentin (Fig. 5). Next, we assessed the effect of HOTAIR knockdown on the expression of following transcription factors known to promote EMT: Snail and Twist. siHOTAIR-transfected cells expressed lower level of snail and twist compared with the siNC-transfected cells (Fig. 5). Collectively, the dysregulation of the expression of EMT-related genes partially explains the involvement of HOTAIR in cervical cancer cell migration and invasion.

\section{Discussion}

In the present study, we found that HOTAIR expression was higher in cervical cancer tissues than in corresponding noncancerous tissues and that it was associated with recurrence in cervical cancer patients. Knockdown of HOTAIR expression decreased cell growth, migration and invasion in cervical cancer cells. The pro-metastatic effects of HOTAIR are likely partially mediated by the regulation of the expression of a number of genes involved in cell migration, invasion and EMT, including VEGF, MMP-9, E-cadherin, $\beta$-catenin, Vimentin, Snail and Twist. Together, our findings suggest that HOTAIR 

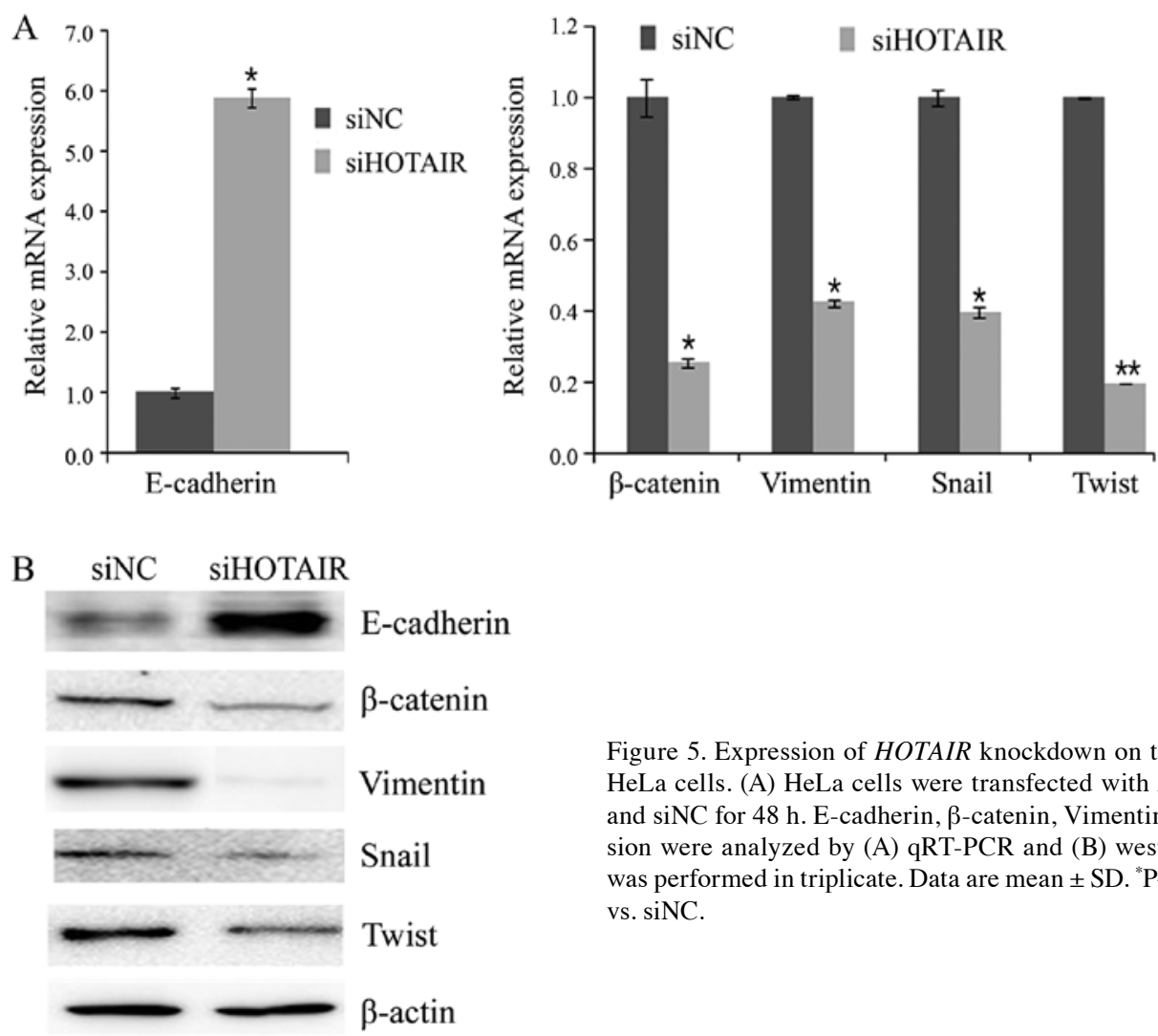

Figure 5. Expression of HOTAIR knockdown on the EMT-related genes in HeLa cells. (A) HeLa cells were transfected with HOTAIR-specific siRNA and siNC for $48 \mathrm{~h}$. E-cadherin, $\beta$-catenin, Vimentin, Snail and Twist expression were analyzed by (A) qRT-PCR and (B) western blotting. Each assay was performed in triplicate. Data are mean \pm SD. ${ }^{*} \mathrm{P}<0.05$ vs. siNC, ${ }^{* *} \mathrm{P}<0.001$ vs. siNC.

may represent a potential biomarker and therapeutic target for cervical cancer.

Although the functional role of small regulatory ncRNAs such as miRNAs in human cancers is now well established, little is known about the regulatory roles of lncRNAs and their relevance to human disease. LncRNAs are transcripts of at least 200 nucleotides without protein-coding potential. Like their protein-coding counterparts, many lncRNAs are capped, spliced and polyadenylated (30). Recent data have shown the tissue-specific expression patterns for lncRNAs. Nevertheless, the growing catalog of functionally characterized lncRNAs reveals that these transcripts are important in different physiological processes $(31,32)$, and therefore, altered expression of lncRNAs may promote cancer development and progression (33). Recently, the IncRNA HOTAIR was associated with metastatic progression in human breast cancer, hepatocellular carcinoma, cervical and pancreatic cancer (16-19). In the present study, HOTAIR expression was associated with disease recurrence in cervical cancer patients and increased the proliferation, migration, and invasion of cervical cancer cells in vitro. Recent reports have shown that lncRNAs are crucial for the regulation of chromatin structure, gene expression and translational control $(34,35)$. However, the detailed functional impact and clinical significance of lncRNA-mediated changes in chromatin and gene expression remain to be elucidated. HOTAIR recruits PRC2 to specific target genes in the genome, which leads to $\mathrm{H} 3 \mathrm{~K} 27$ trimethylation and epigenetic silencing of metastatic suppressor genes (16). Therefore, modifications of DNA-binding proteins by HOTAIR regulates global gene expression. Kogo et al (18) showed that HOTAIR expression was closely correlated with PRC2 occupancy in colorectal cancer patients. Furthermore, in a recent study, HOTAIR-mediated chromatin changes promoted breast cancer metastasis (16). The fact that HOTAIR drives genome-wide chromatin reprogramming suggests that long-range regulation by lncRNAs may be a widespread mechanism. This is supported by a study showing that $>20 \%$ of tested lncRNAs are bound by PRC 2 and other chromatin modifiers (13). These findings provoke questions regarding the initial triggers for HOTAIR overexpression and whether understanding of lncRNA mechanics may have clinical relevance.

The recurrence rate after radical surgery in stage I-II cervical cancer is $\sim 15-30 \%$, and the prognosis of recurrent patients is suboptimal (36). Therefore, identification of reliable biomarkers for predicting recurrence is needed to improve the prognosis of cervical cancer patients. Pelvic lymph node metastasis is the most important postoperative risk factor for recurrence or failure to survive, and thus, cervical cancer patients with metastasis in the pelvic lymph nodes require adjuvant therapy $(21,37,38)$. In the present study, we showed that high HOTAIR expression was correlated with lymph node metastasis and recurrence in cervical cancer. Therefore, analysis of HOTAIR expression in cervical cancer patients may predict the risk of recurrence and, therefore, help guide treatment decisions. Despite the prognostic significance of HOTAIR for tumor recurrence, the results of the present study should be viewed cautiously because of the relatively small sample size. Larger prospective studies are needed to confirm our findings.

HOTAIR has been shown to increase the invasion of many types of cancer cells including pancreatic, breast, colon, and liver cancer cells (16-18). In the present study, we found that 
downregulation of HOTAIR expression decreased cervical cancer cell proliferation, migration and invasion. Therefore, HOTAIR exerts pro-oncogenic activities in cervical cancer and may promote a more aggressive and metastatic phenotype. MMPs play a crucial role in cancer cell invasion and metastasis. MMP-9, which degrades basement membrane collagen, has been shown to promote tumor cell invasion and metastasis and decrease survival in many types of cancer $(29,39)$. It has been generally accepted that tumor angiogenesis plays a critical role in tumor growth, invasion and metastasis. Among the angiogenic factors, VEGF has been shown to have a pivotal role in tumor angiogenesis (40). Knockdown of HOTAIR was associated with reduced expression of VEGF and MMP-9 in BEL7402 hepatocellular carcinoma cells (41). Furthermore, HOTAIR knockdown inhibited proliferation, migration, and invasion through modulation of the extracellular matrix. We also found that downregulation of HOTAIR decreased the expression of VEGF and MMP-9. Taken together, our findings demonstrate that HOTAIR accelerates the aggressiveness of cervical cancer cells through the upregulation of VEGF and MMP-9.

The functional importance of HOTAIR for the activation of invasion indicates that further studies should identify the role of HOTAIR in EMT process (15). It has been demonstrated that knockdown of HOTAIR could reverse EMT process in gastric cancer cells (42). These findings prompted us to determine whether HOTAIR promotes cervical cancer metastasis by regulating the expression of EMT-related genes. As expected, our data suggest that HOTAIR knockdown was dysregulated the expression of EMT-related genes (E-cadherin, $\beta$-catenin, Vimentin, Snail and Twist), implying that these genes participate in HOTAIR-induced cervical cancer metastasis.

In conclusion, our results suggest that HOTAIR is associated with recurrence in cervical cancer. Moreover, HOTAIR may promote cervical cancer progression by inducing cell migration and invasion through the upregulation of VEGF, MMP-9 and expression of EMT-related genes. Thus, HOTAIR may represent a potential therapeutic target and a prognostic marker for cervical cancer.

\section{Acknowledgements}

The present research was supported by the Basic Science Research Program through the National Research Foundation of Korea (NRF) funded by the Ministry of Education, Science and Technology (NRF-2012R1A1A2004523; NRF-2012R1A1A2040271) and the faculty research grant from the Yonsei University College of Medicine for 2014 (6-2014-0025). We thank Dae Ryong Kang and Myeong Hwa Kang for their excellent technical assistance.

\section{References}

1. Guttman M, Amit I, Garber M, et al: Chromatin signature reveals over a thousand highly conserved large non-coding RNAs in mammals. Nature 458: 223-227, 2009.

2. Bernstein E and Allis CD: RNA meets chromatin. Genes Dev 19: 1635-1655, 2005.

3. Mattick JS: The genetic signatures of noncoding RNAs. PLoS Genet 5: e1000459, 2009.

4. Carthew RW and Sontheimer EJ: Origins and mechanisms of miRNAs and siRNAs. Cell 136: 642-655, 2009.
5. Borchert GM, Lanier W and Davidson BL: RNA polymerase III transcribes human microRNAs. Nat Struct Mol Biol 13: 1097-1101, 2006.

6. Pillai RS, Bhattacharyya SN and Filipowicz W: Repression of protein synthesis by miRNAs: how many mechanisms? Trends Cell Biol 17: 118-126, 2007.

7. Berezikov E and Plasterk RH: Camels and zebrafish, viruses and cancer: a microRNA update. Hum Mol Genet 14: R183-R190, 2005.

8. Prasanth KV and Spector DL: Eukaryotic regulatory RNAs: an answer to the 'genome complexity' conundrum. Genes Dev 21: 11-42, 2007.

9. Perez DS, Hoage TR, Pritchett JR, et al: Long, abundantly expressed non-coding transcripts are altered in cancer. Hum Mol Genet 17: 642-655, 2008 .

10. Guttman M, Donaghey J, Carey BW, et al: lincRNAs act in the circuitry controlling pluripotency and differentiation. Nature 477: 295-300, 2011.

11. Rinn JL, Kertesz M, Wang JK, et al: Functional demarcation of active and silent chromatin domains in human HOX loci by noncoding RNAs. Cell 129: 1311-1323, 2007.

12. Ponting CP, Oliver PL and Reik W: Evolution and functions of long noncoding RNAs. Cell 136: 629-641, 2009.

13. Khalil AM, Guttman M, Huarte M, et al: Many human large intergenic noncoding RNAs associate with chromatin-modifying complexes and affect gene expression. Proc Natl Acad Sci USA 106: 11667-11672, 2009.

14. Hung T, Wang Y, Lin MF, et al: Extensive and coordinated transcription of noncoding RNAs within cell-cycle promoters. Nat Genet 43: 621-629, 2011.

15. Gibb EA, Brown CJ and Lam WL: The functional role of long non-coding RNA in human carcinomas. Mol Cancer 10: 38, 2011.

16. Gupta RA, Shah N, Wang KC, et al: Long non-coding RNA HOTAIR reprograms chromatin state to promote cancer metastasis. Nature 464: 1071-1076, 2010.

17. Kim K, Jutooru I, Chadalapaka G, et al: HOTAIR is a negative prognostic factor and exhibits pro-oncogenic activity in pancreatic cancer. Oncogene 32: 1616-1625, 2013.

18. Kogo R, Shimamura T, Mimori K, et al: Long noncoding RNA HOTAIR regulates polycomb-dependent chromatin modification and is associated with poor prognosis in colorectal cancers. Cancer Res 71: 6320-6326, 2011.

19. Huang L, Liao LM, Liu AW, et al: Overexpression of long noncoding RNA HOTAIR predicts a poor prognosis in patients with cervical cancer. Arch Gynecol Obstet 290: 717-723, 2014.

20. Jemal A, Bray F, Center MM, Ferlay J, Ward E and Forman D: Global cancer statistics. CA Cancer J Clin 61: 69-90, 2011.

21. Kodama J, Seki N, Masahiro S, et al: Prognostic factors in stage IB-IIB cervical adenocarcinoma patients treated with radical hysterectomy and pelvic lymphadenectomy. J Surg Oncol 101: 413-417, 2010.

22. Noordhuis MG, Fehrmann RS, Wisman GB, et al: Involvement of the TGF-beta and beta-catenin pathways in pelvic lymph node metastasis in early-stage cervical cancer. Clin Cancer Res 17: 1317-1330, 2011.

23. Kang HW, Wang F, Wei Q, et al: miR-20a promotes migration and invasion by regulating TNKS2 in human cervical cancer cells. FEBS Lett 586: 897-904, 2012.

24. Qiang R, Wang F, Shi LY, et al: Plexin-B1 is a target of miR-214 in cervical cancer and promotes the growth and invasion of HeLa cells. Int J Biochem Cell Biol 43: 632-641, 2011.

25. Liu L, Yu X, Guo X, et al: miR-143 is downregulated in cervical cancer and promotes apoptosis and inhibits tumor formation by targeting Bcl-2. Mol Med Rep 5: 753-760, 2012.

26. Wang $\mathrm{F}$, Li Y, Zhou J, et al: $\mathrm{miR}-375$ is down-regulated in squamous cervical cancer and inhibits cell migration and invasion via targeting transcription factor SP1. Am J Pathol 179: 2580-2588, 2011.

27. Au Yeung CL, Tsang TY, Yau PL and Kwok TT: Human papillomavirus type 16 E6 induces cervical cancer cell migration through the p53/microRNA-23b/urokinase-type plasminogen activator pathway. Oncogene 30: 2401-2410, 2011.

28. Kim KJ, Li B, Winer J, et al: Inhibition of vascular endothelial growth factor-induced angiogenesis suppresses tumour growth in vivo. Nature 362: 841-844, 1993.

29. Curran S and Murray GI: Matrix metalloproteinases: molecular aspects of their roles in tumour invasion and metastasis. Eur J Cancer 36: 1621-1630, 2000. 
30. Carninci P, Kasukawa T, Katayama S, et al: The transcriptional landscape of the mammalian genome. Science 309: 1559-1563, 2005.

31. Dinger ME, Amaral PP, Mercer TR, et al: Long noncoding RNAs in mouse embryonic stem cell pluripotency and differentiation. Genome Res 18: 1433-1445, 2008.

32. Pang KC, Dinger ME, Mercer TR, et al: Genome-wide identification of long noncoding RNAs in $\mathrm{CD}^{+} \mathrm{T}$ cells. J Immunol 182: 7738-7748, 2009.

33. Hall PA and Russell SH: New perspectives on neoplasia and the RNA world. Hematol Oncol 23: 49-53, 2005.

34. Umlauf D, Fraser P and Nagano T: The role of long non-coding RNAs in chromatin structure and gene regulation: variations on a theme. Biol Chem 389: 323-331, 2008.

35. Morris KV and Vogt PK: Long antisense non-coding RNAs and their role in transcription and oncogenesis. Cell Cycle 9 2544-2547, 2010.

36. Kim MK, Jo H, Kong HJ, et al: Postoperative nomogram predicting risk of recurrence after radical hysterectomy for early-stage cervical cancer. Int J Gynecol Cancer 20: 1581-1586, 2010.
37. Soliman PT, Frumovitz M, Sun CC, et al: Radical hysterectomy: a comparison of surgical approaches after adoption of robotic surgery in gynecologic oncology. Gynecol Oncol 123: 333-336, 2011.

38. Biewenga P, van der Velden J, Mol BW, et al: Prognostic model for survival in patients with early stage cervical cancer. Cancer 117: 768-776, 2011.

39. Wiercinska E, Naber HP, Pardali E, van der Pluijm G, van Dam $H$ and ten Dijke P: The TGF-beta/Smad pathway induces breast cancer cell invasion through the up-regulation of matrix metalloproteinase 2 and 9 in a spheroid invasion model system. Breast Cancer Res Treat 128: 657-666, 2011.

40. Burger RA: Role of vascular endothelial growth factor inhibitor in the treatment of gynecologic malignancies. J Gynecol Oncol 21: 3-11, 2010.

41. Geng YJ, Xie SL, Li Q, Ma J and Wang GY: Large intervening non-coding RNA HOTAIR is associated with hepatocellular carcinoma progression. J Int Med Res 39: 2119-2128, 2011.

42. Xu ZY, Yu QM, Du YA, et al: Knockdown of long non-coding RNA HOTAIR suppresses tumor invasion and reverses epithelial-mesenchymal transition in gastric cancer. Int J Biol Sci 9: 587-597, 2013. 